\title{
Chlorquinaldol, a topical agent for skin and wound infections: anti-biofilm activity and biofilm-related antimicrobial cross-resistance
}

This article was published in the following Dove Press journal:

Infection and Drug Resistance

\author{
Alessandro Bidossi $\mathbb{D}^{\prime}$ \\ Marta Bottagisio (D) \\ Roberta De Grandi (D) \\ Lorenzo Drago (D) ${ }^{2}$ \\ Elena De Vecchi (iD \\ 'Laboratory of Clinical Chemistry and \\ Microbiology, IRCCS Orthopedic \\ Institute Galeazzi, Milan, Italy; \\ ${ }^{2}$ Laboratory of Clinical Microbiology, \\ Department of Biomedical Science for \\ Health, University of Milan, Milan, Italy
}

\begin{abstract}
Purpose: Persistence of skin and wound infections is nowadays accepted being linked to bacterial biofilms, which are highly recalcitrant to treatments and contribute to maintain a constant inflammation state and prevent a correct healing. Topical antimicrobials are the most common first-line self-medications; however, treatment failure is not uncommon and emerging resistance to antibiotics is alarming. Chlorquinaldol is an antimicrobial with a wide spectrum of activity and desirable characteristics for topical application. Aim of this study was to evaluate the efficacy of chlorquinaldol to prevent or eradicate $S$. aureus and $P$. aeruginosa biofilms, in comparison to classic topical antibiotics like gentamicin and fusidic acid.
\end{abstract}

Methods: Minimum inhibitory concentrations (MIC) were assessed for each strain and subinhibitory concentrations ( $1 / 2$ and $1 / 4$ MIC) were used in the biofilm assay. Antimicrobial assays were performed during biofilm formation or were applied on mature biofilms and were evaluated by means of crystal violet assay and confocal laser scan microscopy.

Results: Chlorquinaldol and gentamicin were the most effective antimicrobials in both eradicating and preventing pathogens biofilm; however, resistance to methicillin and impermeability to carbapenems impaired chlorquinaldol effect. In addition, similarly to other hydroxyquinolines, aspecific metal chelation is here proposed as chlorquinaldol mode of action.

Conclusion: Relying on an acceptable antibiofilm and a wide spectrum of activity, an aspecific mode of action and consequent absence of resistance development, chlorquinaldol proved to be a good antimicrobial for topical use.

Keywords: chlorquinaldol, biofilm, skin and wound tissue infection, Staphylococcus aureus, Pseudomonas aeruginosa

\section{Introduction}

Skin is the largest organ of the human body, with the main function to protect the inner tissues from invasion of microorganisms of endogenous (skin flora) and exogenous sources. However, in case of skin lesions or wounds, microbes that can evade the host's first lines of defense can adhere to the underlying soft tissues, proliferate and start to produce exopolymeric substances to form a biofilm. ${ }^{1}$ Biofilm is defined as an aggregate community of microbial cells, embedded in a selfproduced slime layer of polymeric substances and adherent to biotic or abiotic surfaces. ${ }^{2}$ Biofilm infections are more commonly found in chronic wounds, which are more likely to be ubiquitous, than in acute wounds ${ }^{3,4}$ and, at first, they do not present with the typical signs of infection and inflammatory symptoms. Once established, bacteria residing in the biofilm are chemically and physically protected
Correspondence: Alessandro Bidossi Laboratory of Clinical Chemistry and Microbiology, IRCCS Orthopedic Institute Galeazzi, Via R. Galeazzi 4, 2016 I Milan, Italy

Tel +39026 62l 4982

Fax +39026 62I 4774

Email

alessandro.bidossi@grupposandonato.it 
from host immune system ${ }^{5}$ and its presence can prolong the inflammatory phase which alters the progression of skin wound healing. Not only biofilm bacteria evade the host defenses, but they are also more recalcitrant to antibiotics and biocides than their planktonic counterparts.

Topical route of administration of antibiotics is usually the first choice for prophylaxis and treatment of uncomplicated skin and wound infections, as it provides high local concentration directly to the site of infections, avoiding systemic side effects due to low absorption. Furthermore, topical formulations of antimicrobials are over-the-counter medications commonly dispensed by community pharmacists. However, there is a lack of evidence for the effectiveness of treatment of established biofilm infections and standardized biofilm susceptibility testing and definition of biofilm-specific breakpoints are needed. ${ }^{6}$ In addition, onset of resistance to topical antimicrobials like fusidic acid in Gram positive bacteria is not uncommon ${ }^{7}$ and levels of resistance to gentamicin are worrisome in particular in Gram negative microorganisms. ${ }^{8}$

Chlorquinaldol (5,7-dichloro-2-methyl-8-quinolinol) has been administered topically for the treatment of skin infections for a long time, alone or in combination with diflucortolone valerate. ${ }^{9-11}$ Its spectrum of activity has been demonstrated for fungal and bacterial species, including Mycobacterium tuberculosis, but also antiviral and antiparasitic effects have been observed; ${ }^{12}$ however, most of these data are more than 30 years old and testing methods might be obsolete. ${ }^{13,14}$ We recently assessed the activity of chlorquinaldol against a collection of bacterial isolates responsible for skin infections, including strains resistant to fusidic acid and gentamicin.8 Staphylococci and enterococci, the most frequent microorganisms causing skin infections, resulted the most sensitive, with inhibitory concentrations considerably lower than those of gentamicin and a higher bactericidal activity in respect to fusidic acid.

The present work was aimed at evaluating the ability to prevent biofilm formation and eradicate established biofilms of Staphylococcus aureus and Pseudomonas aeruginosa, respectively, the most important Gram positive and Gram negative causative agents of skin and wound infections.

\section{Materials and methods Microorganisms}

Clinical strains isolated from skin and wound infections at the IRCCS Orthopedic Institute Galeazzi were chosen from our collection of clinical isolates, routinely isolated in our laboratories after identification and characterization. In particular, biofilm-producing strains of S. aureus - methicillin resistant (MRSA) $(\mathrm{n}=5)$ and susceptible (MSSA) $(\mathrm{n}=5)-$ and $P$. aeruginosa, carbapenems resistant $(\mathrm{n}=5)$ and susceptible $(\mathrm{n}=5)$ were selected. Identification of the isolates was carried out on a Vitek2 Compact (BioMerieux). All strains were stored at $-80^{\circ} \mathrm{C}$ in Brain Heart Infusion broth (BHI, Merck, Darmstadt, Germany) enriched with $10 \%$ glycerol until testing.

\section{Drugs}

Stock solutions of chlorquinaldol (CQ) (MedChemtronica $\mathrm{Ab}$, Sollentuna, Sweden), fusidic acid (FA) (Acros Organics, Geel, Belgium) and gentamicin (GEN) (VWR International Srl, Sanborn, NY, USA) were prepared in 95\% ethanol (CQ) or sterile water (FA and GEN) at concentrations of $5000 \mathrm{mg} / \mathrm{L}$ and stored in aliquots at $-20{ }^{\circ} \mathrm{C}$ until use.

\section{Antimicrobial activity}

The antimicrobial activity was assessed by determining the minimum inhibitory concentration (MIC) by means of broth microdilution method, in accordance with the Clinical and Laboratory Standards Institute guidelines. ${ }^{15}$ Briefly, each bacterial isolate was resuspended in sterile saline to an optical density equal to 0.5 McFarland (approximately $1.5 \times 10^{8} \mathrm{CFU} / \mathrm{mL}$ ), diluted in BHI broth at a final concentration of $5 \times 10^{5} \mathrm{CFU} / \mathrm{mL}$ and inoculated in a sterile 96-wells polystyrene microtiter plate (Biosigma, Cona, Italy) containing a serial 2-fold dilution of CQ, FA or GEN. MIC values, corresponding to the lowest concentration able to inhibit visible bacterial growth, were read after $24 \mathrm{hrs}$ of incubation at $37^{\circ} \mathrm{C}$.

\section{Prevention of biofilm formation}

The ability of the selected antibiotics to prevent $S$. aureus and $P$. aeruginosa biofilm formation was assessed as follows. Biofilm was grown by dispensing $20 \mu \mathrm{L}$ aliquots of overnight broth cultures with an optical density equal to $0.5 \mathrm{McF}$ arland standards $\left(1.5 \times 10^{8} \mathrm{CFU} / \mathrm{mL}\right)$ into a sterile 96-wells polystyrene microtitre plate containing $180 \mu \mathrm{L}$ of BHI alone (positive controls) or $180 \mu \mathrm{L}$ of BHI containing sub-inhibitory concentrations $(1 / 2 \times$ MIC and $1 / 4 \times$ MIC) of the tested antibiotics. After incubation for $72 \mathrm{hrs}$ at $37{ }^{\circ} \mathrm{C}$, broth was removed, together with non-adherent bacteria, and the amount of biofilm was evaluated by a spectrophotometric assay, as described below. The experiment was performed in triplicate for each tested strain. 


\section{Interference on pre-formed biofilm}

To investigate whether the tested antibiotics were able to break down existing biofilm, biofilm of each strain was grown for $72 \mathrm{hrs}$, as described above, and then incubated for additional $72 \mathrm{hrs}$ in the presence of sub-inhibitory concentrations ( $1 / 2 \mathrm{MIC}$ and $1 / 4 \mathrm{MIC})$ of the tested antibiotics. At the end of the treatment, the amount of biofilm was evaluated by means of a spectrophotometric assay, as described below, and expressed as percentage in respect to pre-treatment biofilm. The experiment was performed in triplicate for each strain.

\section{Spectrophotometric assay}

The amount of biofilm was determined by adopting the spectrophotometric assay developed by Christensen et al ${ }^{16}$. Briefly, culture medium was removed at the end of the incubation time, and two washes with saline were performed in order to remove non-adherent bacteria. After air-drying, each well was stained with $1 \%$ crystal violet solution (Merck Darmstadt, Germany) for 10 mins and the dye in excess was removed with three washes with saline. Once dried, absolute ethanol was added to each well to solubilize the dye attached to the biofilm. The amount of biofilm was determined by spectrophotometric reading at $595 \mathrm{~nm}$ wavelength using a microplate reader (Multiskan FC; ThermoFisher Scientific, Ratastie, Finland).

\section{Confocal laser scanning microscopy (CLSM) assay}

For CLSM analysis, biofilms were cultured using the same setup described above, adjusting reagents volumes for 24-well plates (Biosigma, Cona, Italy). Each well contained a sterile 10-mm diameter glass microscope coverslip (VWR International Srl, Sanborn, NY, USA). Biofilms were gently washed with sterile saline and stained with Filmtracer ${ }^{\mathrm{TM}}$ LIVE/DEAD ${ }^{\mathrm{TM}}$ Biofilm Viability Kit (Molecular Probes, Inc, Eugene, OR, USA), adjusting the manufacturer's instructions. Briefly, the staining solution was prepared by adding 1.5 $\mu \mathrm{L}$ of SYTO9 and $1.5 \mu \mathrm{L}$ of propidium iodide to $1 \mathrm{~mL}$ of filter-sterilized water. Biofilm samples were stained by incubation with $20 \mu \mathrm{L}$ of staining solution for 15 mins in the dark. After incubation, samples were washed with saline and examined with an upright TCS SP8 (Leica Microsystems CMS GmbH, Wetzlar, Germany) using a $20 \times$ dry objective (HC PL FLUOTAR 20×/0.50). A 488-nm laser line was used to excite SYTO9, while a 552-nm laser line was used to excite propidium iodide stain. Images from at least three randomly selected areas were acquired for each coverslip. The obtained images were processed with Las X (Leica Microsystems CMS $\mathrm{GmbH}$, Wetzlar, Germany) and analyzed with Fiji software (Fiji, ImageJ, Wayne Rasband National Institutes of Health).

\section{$P$. aeruginosa pigment production quantification}

Pyocianin, aeruginosin A and pyoverdin production by $P$. aeruginosa were assessed spectrophotometrically. Briefly, culture media from biofilm assays grown in 24-well microplate were collected, centrifuged and the supernatants filtered through a $0.2-\mu \mathrm{m}$ filter. Successively, the supernatants were placed in $1 \mathrm{~mL}$ cuvettes and read at $402 \mathrm{~nm}$ to assess pyoverdin production, ${ }^{17}$ at $695 \mathrm{~nm}$ for pyocianin production ${ }^{18}$ and at $512 \mathrm{~nm}$ for aeruginosin A production. ${ }^{19}$

\section{Statistical analysis}

Results were expressed as mean \pm standard deviation and analyzed for statistical significance with PRISM5 software (GraphPad Software, San Diego, CA, USA) using oneway analysis of variance (ANOVA) followed by Bonferroni post-hoc correction. A $P$-value $\leq 0.05$ was used as the significance level.

\section{Results}

\section{Evaluation of minimum inhibitory concentration (MIC)}

Table 1 shows the MIC values of the tested antibiotics for $S$. aureus and $P$. aeruginosa selected strains. CQ concentrations able to inhibit all the $S$. aureus strains fell in a range between 0.125 and $1 \mathrm{mg} / \mathrm{L}$. All the strains presented a MIC value of $1 \mathrm{mg} / \mathrm{L}$ for GEN, made exception for strain 5 , which had a MIC value of $0.5 \mathrm{mg} / \mathrm{L}$, while FA displayed MIC values ranging between 0.004 and $0.125 \mathrm{mg} / \mathrm{L}$.

CQ MIC values for $P$. aeruginosa ranged between 32 and $256 \mathrm{mg} / \mathrm{L}$, while those for GEN were between 1 and $4 \mathrm{mg} / \mathrm{L}$.

\section{Evaluation of biofilm inhibition Spectrophotometric assay} Prevention of staphylococcal biofilm formation

FA displayed a very low activity in preventing biofilm formation of both MRSA and MSSA strains, while GEN displayed a significant reduction of biofilm biomass of resistant and susceptible strains at all the tested concentration. Finally, CQ showed the highest activity against MSSA, while a limited reduction of MRSA biofilm was 
Table I Minimum inhibitory concentration

\begin{tabular}{|c|c|c|c|}
\hline & Chlorquinaldol & Gentamicin & Fusidic acid \\
\hline \multicolumn{4}{|l|}{ S. aureus } \\
\hline Sau I (S) & 0.25 & I & 0.004 \\
\hline Sau 2 (S) & 0.25 & I & 0.016 \\
\hline Sau 3 (S) & 0.25 & I & 0.032 \\
\hline Sau 4 (S) & 0.25 & I & 0.008 \\
\hline Sau 5 (S) & 0.25 & 0.5 & 0.125 \\
\hline Sau $6(R)$ & 0.25 & I & 0.008 \\
\hline Sau 7 (R) & 1 & I & 0.25 \\
\hline Sau 8 (R) & 0.5 & 1 & 0.064 \\
\hline Sau 9 (R) & 0.125 & I & 0.125 \\
\hline Sau 10 (R) & 0.125 & I & 0.25 \\
\hline \multicolumn{4}{|c|}{ P. aeruginosa } \\
\hline & Chlorquinaldol & Gentamicin & \\
\hline Pae I (S) & 64 & 2 & \\
\hline Pae 2 (S) & 32 & 4 & \\
\hline Pae 3 (S) & 256 & 2 & \\
\hline Pae 4 (S) & 256 & 2 & \\
\hline Pae 5 (S) & 256 & I & \\
\hline Pae 6 (R) & 32 & 2 & \\
\hline Pae 7 (R) & 64 & 4 & \\
\hline Pae 8 (R) & 256 & 2 & \\
\hline Pae 9 (R) & 128 & 2 & \\
\hline Pae $10(\mathrm{R})$ & 256 & 2 & \\
\hline
\end{tabular}

Notes: Data are expressed in $\mathrm{mg} / \mathrm{L}$. Pae, P. aeruginosa, Sau, S. aureus, S, methicillin susceptible $S$. aureus or carbapenems susceptible $P$. aeruginosa, $R$, methicillin resistant $S$. aureus or carbapenems resistant $P$. aeruginosa.

observed at both the tested concentrations (Figure 1A). In fact, while GEN and FA effect on MRSA and MSSA biofilm did not differ, a significant difference between methicillin susceptible and resistant strains could be observed for CQ (Table 2).

\section{Treatment of staphylococcal pre-formed biofilm}

All the tested antibiotics showed a significant activity on the eradication of a preformed biofilm of MSSA, while only GEN and, to a lesser extent, FA were effective against MRSA biofilm (Figure 1B). Similarly to the data obtained on biofilm formation, a significant difference in activity of CQ was observed against pre-formed biofilm produced by MRSA and MSSA (Figure 1B).

\section{Prevention of $P$. aeruginosa biofilm formation}

Only $1 / 2$ MIC concentration of GEN were able to significantly prevent formation of $P$. aeruginosa biofilm by both susceptible and, to a lesser extent, resistant strains (Figure 1C).

\section{Treatment of $P$. aeruginosa pre-formed biofilm}

Both CQ and GEN displayed a high efficacy in eradicating established biofilms of $P$. aeruginosa strains susceptible to carbapenems (Figure 1D). On the contrary, only the highest concentrations of GEN were able to significantly reduce resistant $P$. aeruginosa biofilm. Similarly to what was observed for $S$. aureus, CQ treatment of resistant $P$. aeruginosa biofilm was significantly less effective than that of the susceptible counterpart; however, also GEN revealed an impaired effect on resistant strain, in particular with lower concentrations (Table 2).

\section{CLSM analysis}

\section{Prevention of staphylococcal biofilm formation}

MSSA biofilm forming cells were heavily affected by the presence of sub-inhibitory concentrations of CQ and GEN, while no effects could be observed by the addiction of FA (Figures 2A and 4). By contrast, MRSA biofilm could only be significantly inhibited by the highest concentration of GEN (Figure 2A). The highest mortality of MSSA cells in biofilm formation was induced by the highest concentrations of gentamicin $(25.3 \%)$, while chlorquinaldol displayed the highest killing rate of MRSA cells (25.4\%), which is also clearly appreciable in the three-dimensional reconstruction (Figure 4). Generally, fusidic acid was the least effective in killing biofilm forming cells (Table 3).

\section{Treatment of staphylococcal pre-formed biofilm}

Similarly to what was observed in biofilm forming cells, only the addition of CQ and GEN to mature biofilm drastically reduced the viability of cells (Figures $2 \mathrm{~B}$ and 4). Again, the highest killing rate on MSSA was induced by GEN and by CQ in MRSA, while FA was the least effective both in reducing the number of cells in a mature biofilm and in the live/dead cells ratio (Figures 2B and 4, Table 3).

\section{Prevention of $P$. aeruginosa biofilm formation}

CQ was able to inhibit biofilm formation by $P$. aeruginosa susceptible to carbapenems at a higher extent with respect to GEN; however, it proved to be ineffective against the resistant strains (Figure 2C). On the other hand, only CQ was able to increase the killing rate of both susceptible and resistant $P$. aeruginosa (Table 3). When analyzing the three-dimensional reconstructing of the treated and untreated biofilms, it could be noted that both the antimicrobials acted by reducing the biofilm burden, while leaving the biofilm shape similar to that of the control (Figure 5). 
A



C

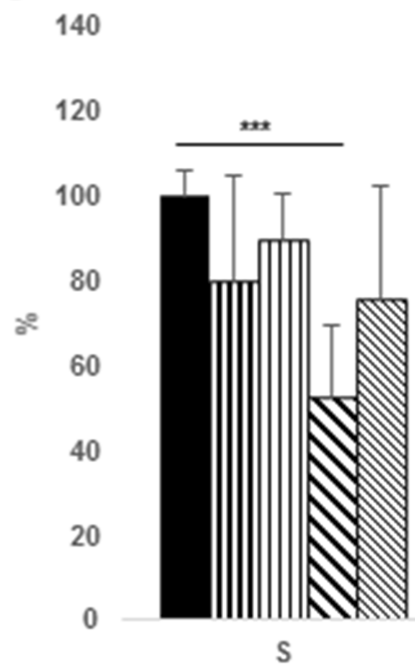

Ctrl

四 $C Q \frac{1}{2} M I C$

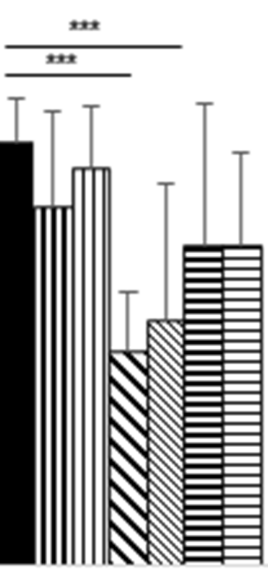

$\mathrm{R}$

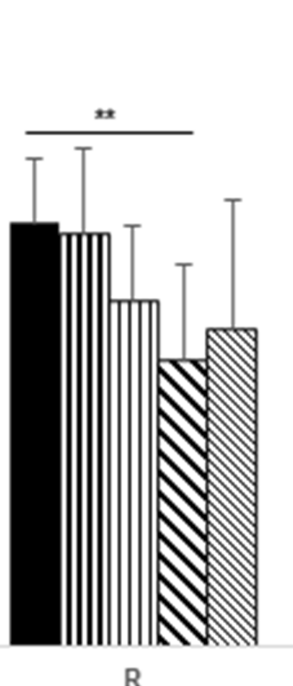

B

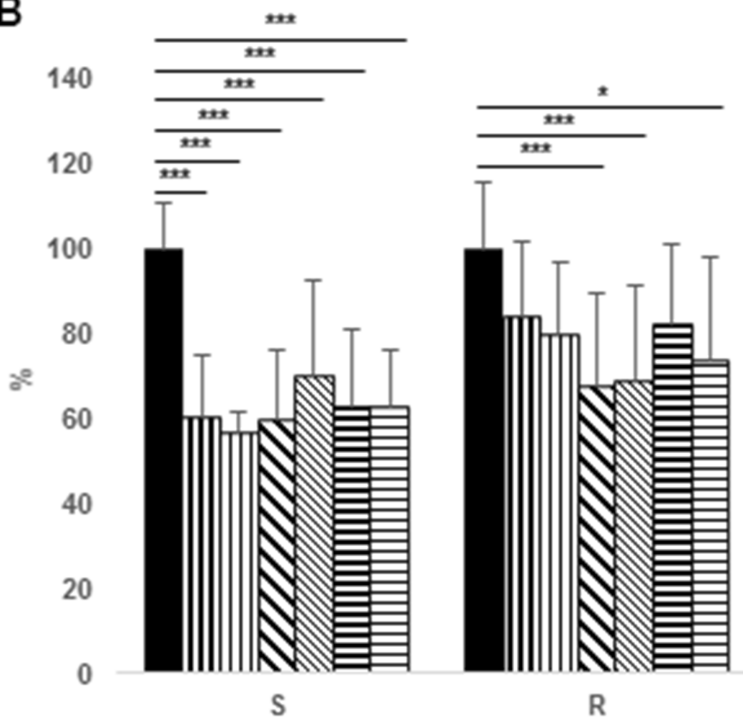

D
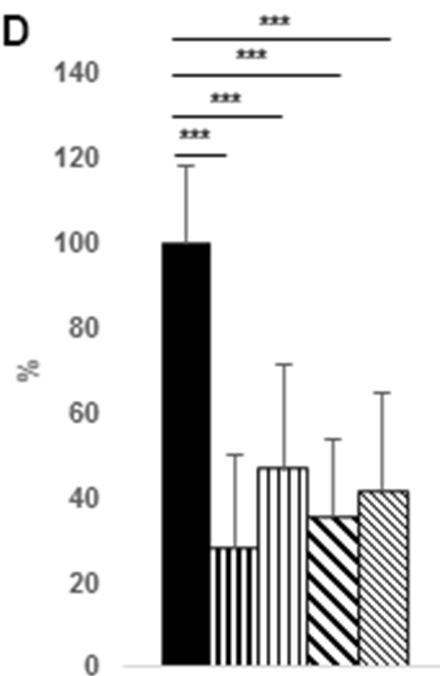

$\mathrm{s}$

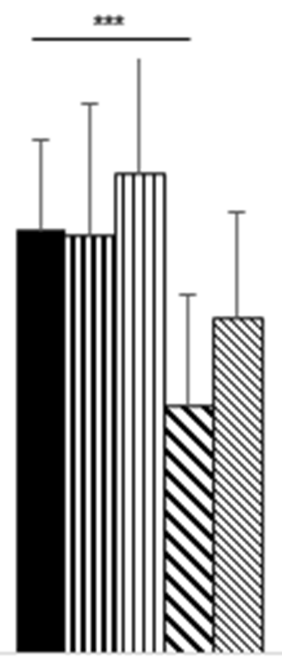

$\mathrm{R}$

血 $C Q 1 / 4 \mathrm{MIC}$

GM $1 \frac{1}{2} M I C$

$\mathbb{Q}$ GM $1 / 4 M I C \quad$ 目 AF $1 / 4 M I C$

AF $1 / 4$ MIC

Figure I Effect of antimicrobial treatment on $S$. aureus and $P$. aeruginosa biofilm analyzed by means of crystal violet assay. Data are expressed as percentage reduction with respect to control. (A) Prevention of S. aureus biofilm formation. (B) Treatment of S. aureus pre-formed biofilm. (C) Prevention of $P$. aeruginosa biofilm formation. (D) Treatment of $P$. aeruginosa pre-formed biofilm. $S=$ MSSA or $P$. aeruginosa susceptible to carbapenems, $R=$ MRSA or $P$. aeruginosa resistant to carbapenems. Ctrl, control (black bars), CQ, chlorquinaldol ( $1 / 2$ MIC, bars with thicker vertical lines, $1 / 4$ MIC, bars with thinner vertical lines), GEN, gentamicin ( $1 / 2$ MIC, bars with thicker oblique lines, $1 / 4$ MIC, bars with thinner oblique lines), FA, fusidic acid ( $1 / 2$ MIC, bars with thicker horizontal lines, $1 / 4$ MIC, bars with thinner horizontal lines). $*_{p}<0.01$, $* * p<0.005$, $* * * p<0.00 \mathrm{I}$.

\section{Treatment of $P$. aeruginosa pre-formed biofilm}

Treatment of a mature $P$. aeruginosa biofilm displayed similar results to those observed with treatment on growing biofilm. Both the antibiotics were effective against the susceptible strains at all the tested concentrations, while only GEN was able to exert a significant reduction of resistant strains (Figure 2D). Killing rate of the two tested antimicrobials was similar and only slightly increased with respect to the control (Table 3).

\section{Pigments production}

Chlorquinaldol and gentamicin displayed a strong inhibition of aeruginosin A production at all the tested concentrations, while no effect could be observed on pyoverdin. Interestingly, only chlorquinaldol was able to inhibit pyocyanin production (Figure 3).

\section{Discussion}

The association of microbial biofilms to chronicity of infected wounds is today widely accepted. ${ }^{3,4}$ These densely aggregated 
Table 2 Statistically significant differences between susceptible and resistant strains

\begin{tabular}{|c|c|c|c|c|}
\hline & \multicolumn{4}{|l|}{ Crystal violet assay } \\
\hline & \multicolumn{2}{|l|}{ MRSA vs MSSA } & \multicolumn{2}{|l|}{ P. aeruginosa $R$ vs $S$} \\
\hline & $\begin{array}{l}\text { Prevention of biofilm } \\
\text { formation }\end{array}$ & $\begin{array}{l}\text { Treatment of pre-formed } \\
\text { biofilm }\end{array}$ & $\begin{array}{l}\text { Prevention of biofilm } \\
\text { formation }\end{array}$ & $\begin{array}{l}\text { Treatment of pre-formed } \\
\text { biofilm }\end{array}$ \\
\hline $\mathrm{CQ} 1 / 2 \mathrm{MIC}$ & $p<0.005$ & $p<0.01$ & - & $p<0.001$ \\
\hline $\mathrm{CQ} 1 / 4 \mathrm{MIC}$ & $p<0.01$ & $p<0.01$ & - & $p<0.001$ \\
\hline GEN $1 / 2$ & - & - & - & - \\
\hline \multirow{8}{*}{$\begin{array}{l}\text { MIC } \\
\text { GEN } 1 / 4 \\
\text { MIC } \\
\text { FA } 1 / 2 \text { MIC } \\
\text { FA } 1 / 4 \text { MIC }\end{array}$} & & & & \\
\hline & - & - & - & $p<0.005$ \\
\hline & & & & \\
\hline & - & - & & \\
\hline & - & - & & \\
\hline & \multicolumn{4}{|l|}{ CLSM assay } \\
\hline & \multicolumn{2}{|l|}{ MRSA vs MSSA } & \multicolumn{2}{|l|}{ P. aeruginosa $\mathrm{R}$ vs $\mathrm{S}$} \\
\hline & $\begin{array}{l}\text { Prevention of biofilm } \\
\text { formation }\end{array}$ & $\begin{array}{l}\text { Treatment of pre-formed } \\
\text { biofilm }\end{array}$ & $\begin{array}{l}\text { Prevention of biofilm } \\
\text { formation }\end{array}$ & $\begin{array}{l}\text { Treatment of pre-formed } \\
\text { biofilm }\end{array}$ \\
\hline $\mathrm{CQ} 1 / 2 \mathrm{MIC}$ & $p<0.01$ & $p<0.01$ & $p<0,001$ & $p<0.001$ \\
\hline $\mathrm{CQ} 1 / 4 \mathrm{MIC}$ & $p<0.01$ & - & - & $p<0.005$ \\
\hline GEN $1 / 2$ & - & - & - & - \\
\hline MIC & & & & \\
\hline GEN $1 / 4$ & - & - & - & - \\
\hline MIC & & & & \\
\hline FA $1 / 2$ MIC & - & - & & \\
\hline FA $1 / 4 \mathrm{MIC}$ & - & - & & \\
\hline
\end{tabular}

Notes: $S, P$. aeruginosa susceptible to carbapenems, R, P. aeruginosa resistant to carbapenems.

Abbreviations: CLSM, Confocal laser scanning microscopy; CQ, chlorquinaldol; GEN, gentamicin; FA, fusidic acid; MRSA, methicillin resistant Staphylococcus aureus; MSSA, methicillin susceptible Staphylococcus aureus.

microbial cells encased in an extracellular matrix represent a physical barrier to wound healing, maintaining a status of constant inflammation thereby causing more damage and delaying the epithelialization of the tissues. In addition, chronic inflammatory skin disorders have been reported to be more susceptible to colonization by pathogens biofilms, which in turn contribute to the inflammatory state. ${ }^{20-22}$ Biofilm-associated infections are hardly resolved, even in immunocompetent patients; however, administration of antibiotics for topical use showed to be beneficial in the treatment of skin infections and to facilitate the healing especially when combined with anti-inflammatory drugs. ${ }^{20}$ Topical route permits the application of high concentrations of antibacterial agent directly at the site of infection, with low incidence of systemic side effects.

In an era where increasing antibiotic resistance and appearance of multidrug-resistant bugs represent a serious cause of concern and development of new antibiotics is drastically slowing down, old antimicrobials are increasingly re-proposed. ${ }^{23}$ Recently, we tested the 8-hydroquinoline (8HQ) derivative chlorquinaldol for its ability to inhibit Gram positive and Gram negative clinical isolates of skin infections. ${ }^{8} \mathrm{CQ}$ can be found in the form of a cream usually in combinations with the corticosteroid diflucortolone valerate and its use was found to be effective for a wide range of cutaneous disease of allergic or infective nature, both in vivo and in vitro, and skin reactions were observed to be very uncommon. ${ }^{9-11,14,24-27}$ Despite the promising characteristic, this topical agent entered the market just when antibiotic development was in its golden age and slowly fell into disuse.

In our previous work, broth microdilution and killing curves in comparison with GEN and FA revealed interesting features of CQ. Its most prominent activity is against staphylococci (with no differences between MRSA and MSSA), with a more pronounced bactericidal activity FA and lower inhibitory concentrations than GEN, for which a high incidence of resistant strains was observed (80\%). Concomitantly, in a recent study where 1524 compounds from a clinical 

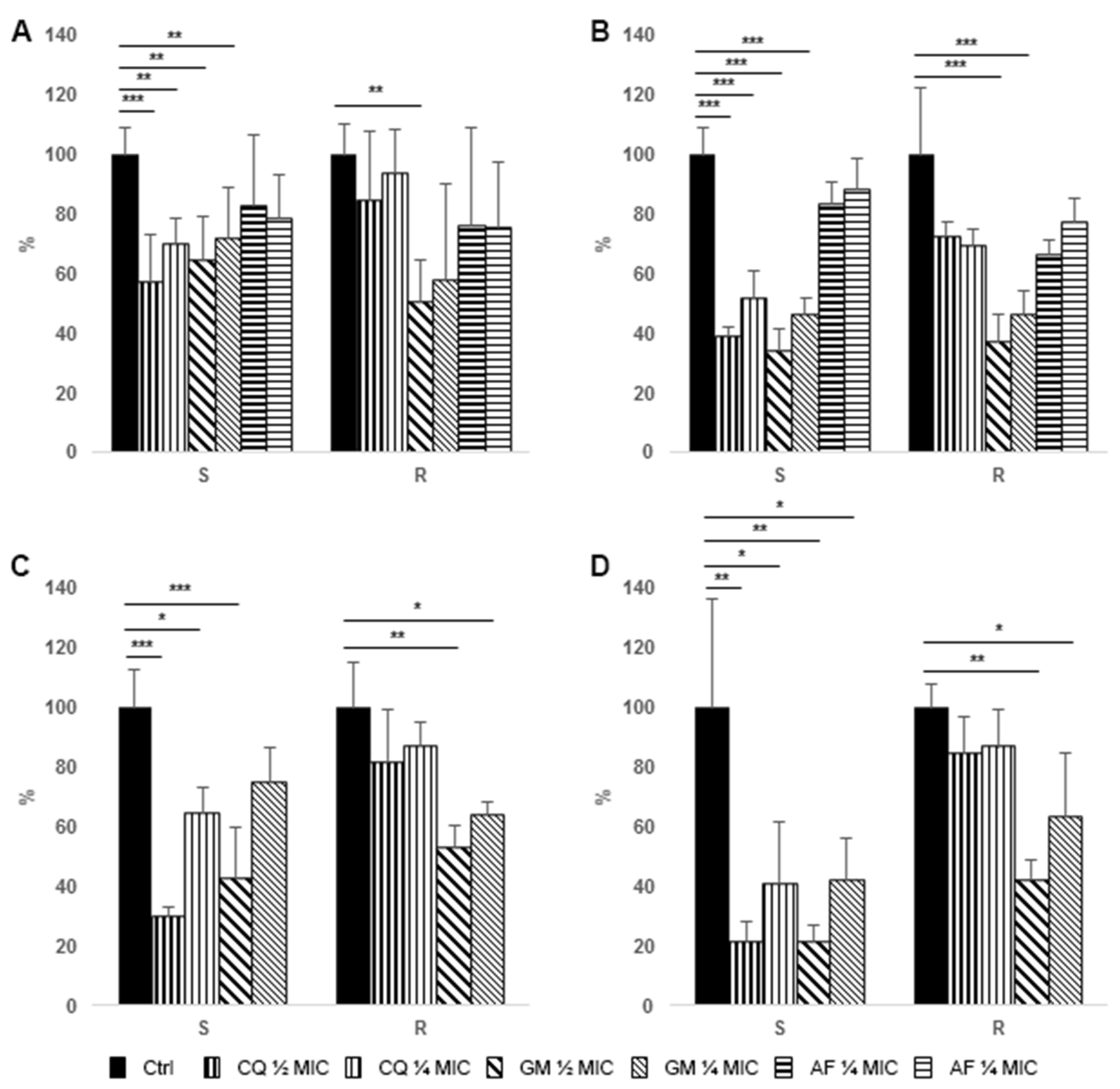

Figure 2 Effect of antimicrobial treatment on S. aureus and $P$. aeruginosa biofilm analyzed by means of confocal laser scan microscopy. Data are expressed as percentage reduction with respect to control. (A) Prevention of $S$. aureus biofilm formation. (B) Treatment of $S$. aureus pre-formed biofilm. (C) Prevention of $P$. aeruginosa biofilm formation. (D) Treatment of $P$. aeruginosa pre-formed biofilm. $S=$ MSSA or $P$. aeruginosa susceptible to carbapenems, $R=M R S A$ or $P$. aeruginosa resistant to carbapenems. Ctrl, control (black bars), CQ, chlorquinaldol ( $1 / 2$ MIC, bars with thicker vertical lines, $1 / 4$ MIC, bars with thinner vertical lines), GEN, gentamicin ( $1 / 2$ MIC, bars with thicker oblique lines, $1 / 4$ MIC, bars with thinner oblique lines), FA, fusidic acid $(1 / 2 \mathrm{MIC}$, bars with thicker horizontal lines, $1 / 4 \mathrm{MIC}$, bars with thinner horizontal lines). $* p<0.0 \mathrm{I}$. $* * p<0.005, * * * p<0.001$.

compound library were tested on an MRSA strain, CQ was indicated among the most active molecules assayed. ${ }^{27}$

However, classical routes of antimicrobial testing might fail in predicting efficacy against biofilm-associated infections, reflecting the failure of conventional antibiotics and explaining the consistent emergence of resistance patterns. Different mechanisms have been hypothesized to explain the reduced activity of some antibiotics against bacteria forming biofilm. A slow penetration through the biofilm matrix may give time to the cells to adapt phenotypically. Uptake of exogenous DNA, horizontal gene transfer and spontaneous mutations have been largely described being very frequent in sessile cells ${ }^{28-33}$ and might explain the emerging resistance to fusidic acid correlated to its topical use. ${ }^{7,34}$ Hence, there is urgent need to provide more specific information on the ability of classic and new antimicrobials to prevent or eradicate biofilms.

In this work, we tested CQ efficacy against biofilm of $S$. aureus and $P$. aeruginosa, respectively, the most representative Gram positive and Gram negative causative 


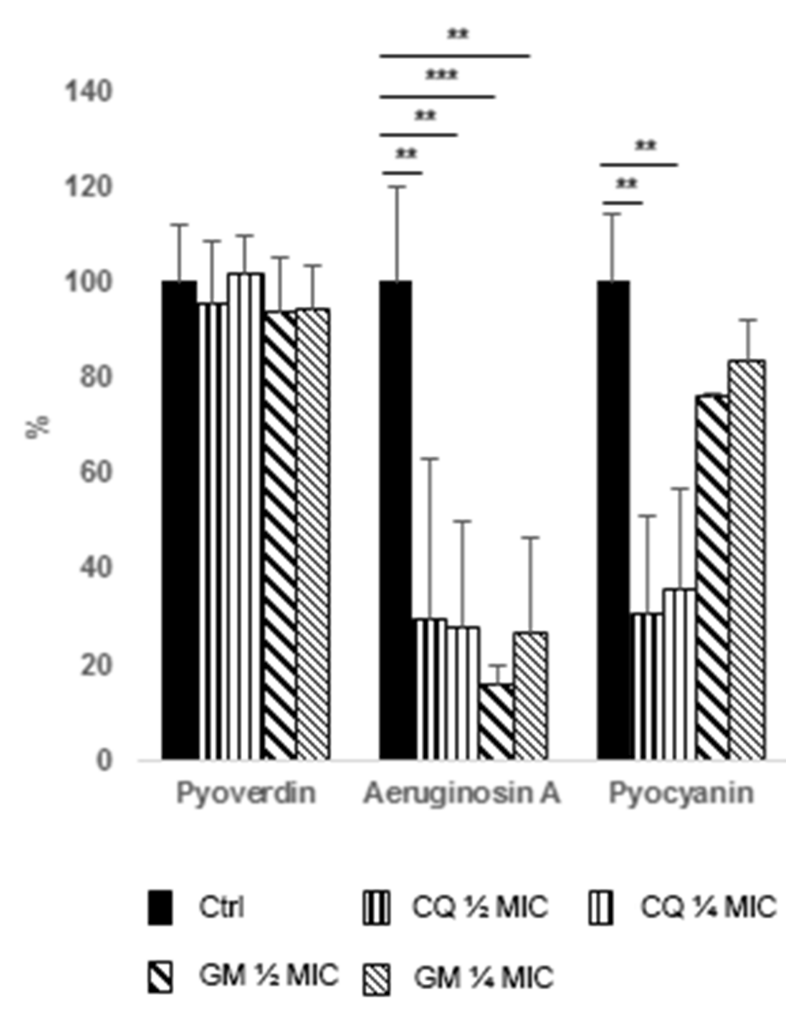

Figure 3 Effect of antimicrobial treatment on pigment production by $P$. aeruginosa. Data are expressed as percentage reduction with respect to control. Ctrl, control (black bars), CQ, chlorquinaldol ( $1 / 2 \mathrm{MIC}$, bars with thicker vertical lines, $1 / 4 \mathrm{MIC}$, bars with thinner vertical lines), GEN, gentamicin ( $1 / 2$ MIC, bars with thicker oblique lines, $1 / 4$ MIC, bars with thinner oblique lines). $* p<0.01, * * p<0.005, * * *<<.001$.

agents of skin and wound infections and among the major biofilm producer bacteria, in comparison to GEN and FA, two of the most used topical antimicrobials. Initially, we randomly chose five strains each of $S$. aureus and $P$. aeruginosa from our clinical collection, isolated from skin or wound infections. However, though MIC values fell in a narrow range of concentrations (Table 1), a heterogeneous effect of CQ could be observed (data not shown). Indeed, a lack of effectiveness was found only for methicillin-resistant staphylococci and for carbapenems-resistant $P$. aeruginosa (Table S1). Therefore, we decided to proceed by adding five more isolates to both the species, so to have a group of five resistant and five susceptible isolates each. The results initially obtained were maintained for the two species when biofilm inhibition or biofilm eradication were analyzed both with crystal violet or CLSM assay (Figures 1 and 2).

S. aureus biofilm formation inhibition and mature biofilm eradication by GEN and FA was similar in MSSA and MRSA, with GEN always displaying a significant effect and FA having limited or no efficacy (Figures 1 and 2). On the other hand, CQ treatment of MSSA was definitely comparable to that of
GEN, while its effect was markedly impaired when MRSA strains were tested (Figures 1 and 2). We hypothesized that the reason for this behavior could be related to the different mechanisms of biofilm formation and matrix composition of MSSA and MRSA. Acquisition of SCCmec element was found to significantly impact on global gene regulation and, in particular, on cell wall architecture and biofilm formation. ${ }^{35}$ While MSSA biofilm formation mostly relies on ica-dependent production of Polysaccharide Intercellular Adhesin, ica locus was found to be redundant in MRSA, for which matrix is held together by a dense net of protein adhesins and eDNA. $^{36,37}$ Along with changes in matrix composition, a dramatic downregulation of virulence factors was observed in MRSA; nevertheless, no differences were observed in terms of biofilm biomass production and ability to colonize surfaces. ${ }^{36,37}$ Interestingly, MRSA treated with CQ displayed a higher dead cell ratio in comparison to MSSA, while GEN was more bactericidal toward MRSA strains (Table 3).

Similar results were obtained for $P$. aeruginosa, with isolates resistant to carbapenems being more recalcitrant to CQ treatment. Subinhibitory concentrations of CQ were unable to display a significant effect also against susceptible strains during biofilm formation when analyzed with crystal violet assay (Figure 1A), while a drastic reduction could be observed on CLSM assay (Figure 2A). However, more consistent reductions were generally observed in CLSM assay with respect to crystal violet staining. Indeed, while the first permit to detect only the biomass of the cells embedded in the biofilm, crystal violet might be able to aspecifically stain all organic compounds present, matrix included, if not removed by a solvent as in Gram staining. In fact, up to $90 \%$ of the biofilm mass is composed of extracellular substances ${ }^{38}$ and cell reduction by a treatment might be masked when analyzed with crystal violet assay. On the other hand, these results highlight the fact that both the antimicrobials exerted their action more on cell viability than on matrix production, thus leaving a biofilm architecture resembling that of the untreated control (Figures 4 and 5).

Notably, during the biofilm assay, we noted that some $P$. aeruginosa strains were producing large amounts of pigments and that subMIC concentrations of antibiotics were able to inhibit their secretion into the medium, with no differences between resistant and susceptible strains. Thus, we collected the supernatants from the wells to assess the production of the most common $P$. aeruginosa pigments. While production of pyoverdin, a known siderophore for iron scavenging, ${ }^{39}$ was not impaired, aeruginosin A and pyocyanin showed reduction in presence of 


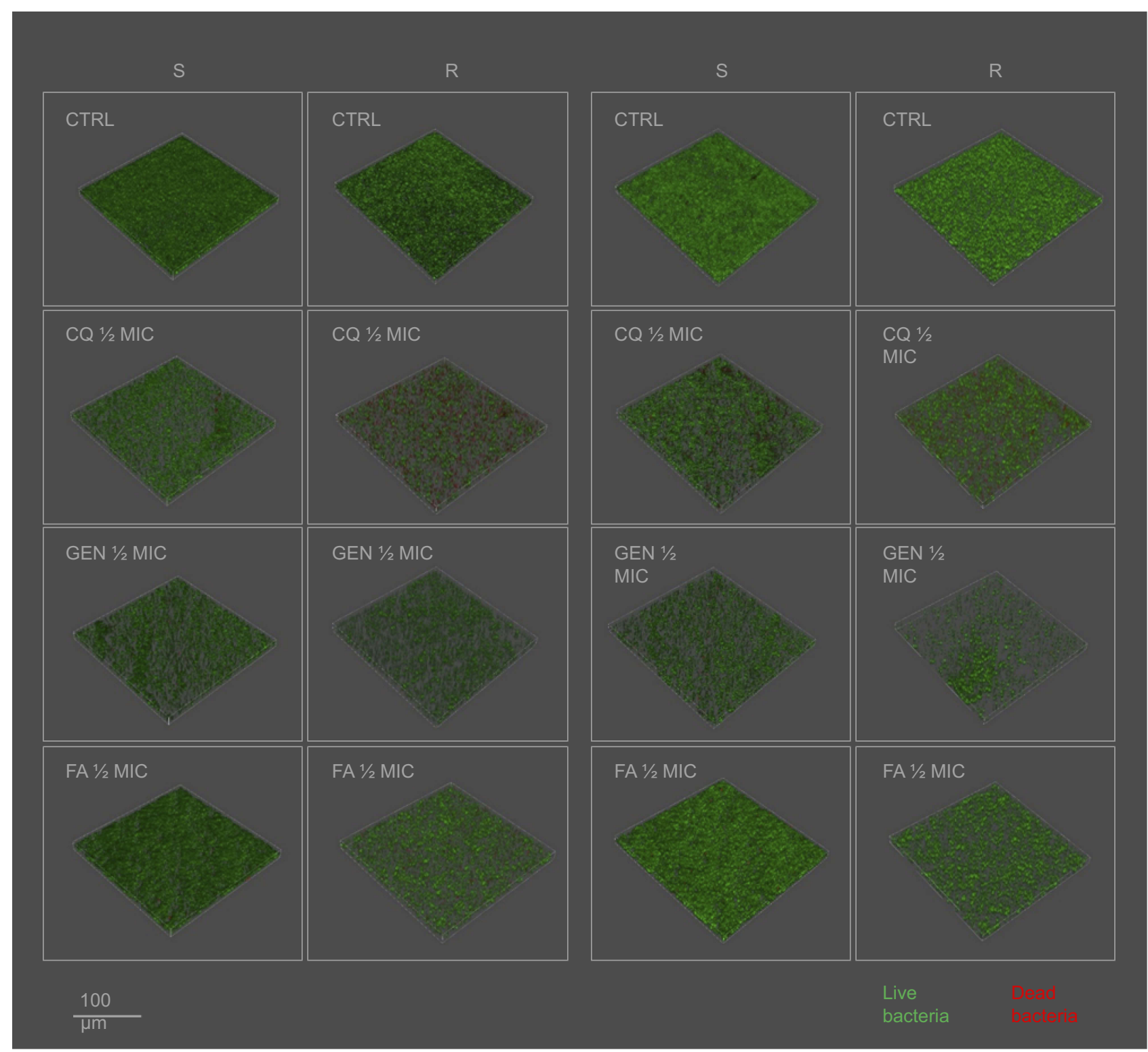

Figure 4 Three-dimensional reconstruction of $S$. aureus biofilm analyzed by means of confocal laser scan microscopy. Abbreviations: S, MSSA; R, MRSA; CQ, chlorquinaldol; GEN, gentamicin; FA, fusidic acid.

both CQ and GEN (Figure 3). Aeruginosin A is a red pigment produced after prolonged aerobic growth, with important reducing properties. ${ }^{19}$ Pyocyanin is a blue phenazine compound and a renowned $P$. aeruginosa virulence factor with multiple roles. It is known to exert cytotoxic and antibacterial action, inhibiting cellular respiration and production of oxygen radicals, to promote inflammation processes and to serve as a signaling molecule in $P$. aeruginosa quorum sensing. ${ }^{18,40}$ Furthermore, pyocyanin was found to increase the incorporation of eDNA in the biofilm matrix by direct binding, thus improving cellular aggregation. $^{41}$ While aeruginosin A production was reduced of $70-85 \%$ by both the antibiotics, pyocyanin was significantly reduced only by CQ (Figure 3), possibly meaning a specific inhibition. CQ, 8HQ and other 8HQ derivatives are known to possess diverse chemical and biological activities, which are a result of their ability to chelate multiple metal ions. ${ }^{12,42}$ In fact, different are the processes by which $8 \mathrm{HQ}$ and derivatives are proposed to exert bactericidal activity. Data from electron microscopy indicated that $8 \mathrm{HQ}$ actively disrupts $S$. aureus cell walls, leading to cell lysis, ${ }^{43}$ or it is proposed that hydroxyquinolines take advantage of their lipophilicity to penetrate bacterial cell membranes in order to reach its target site of 
Table 3 Dead cell percentage in biofilm

\begin{tabular}{|c|c|c|c|c|c|c|c|c|}
\hline & & Control & CQ $1 / 2$ MIC & CQ $1 / 4 \mathrm{MIC}$ & GEN $1 / 2$ MIC & GEN $1 / 4$ MIC & FA $1 / 2$ MIC & FA $1 / 4$ MIC \\
\hline \multicolumn{9}{|l|}{ S. aureus } \\
\hline \multirow[t]{2}{*}{ Treatment of biofilm formation } & $\mathrm{S}$ & 5.12 & 13.47 & 10.60 & 25.28 & $|4.5|$ & 2.95 & 3.01 \\
\hline & $\mathrm{R}$ & 10.48 & 25.37 & 19.49 & 7.88 & 7.64 & 7.02 & 9.61 \\
\hline \multirow[t]{2}{*}{ Treatment of pre-formed biofilm } & $\mathrm{S}$ & 10.08 & 7.67 & 6.38 & $19.7 \mid$ & 21.64 & 13.28 & 15.42 \\
\hline & $\mathrm{R}$ & 6.29 & 11.54 & 19.79 & 9.87 & 9.98 & 9.85 & 6.29 \\
\hline \multicolumn{9}{|l|}{ P. aeruginosa } \\
\hline \multirow[t]{2}{*}{ Treatment of biofilm formation } & $\mathrm{S}$ & 9.67 & 18.94 & 17.49 & 9.23 & 6.73 & & \\
\hline & $\mathrm{R}$ & 4.36 & 15.49 & 20.94 & 3.35 & 1.51 & & \\
\hline \multirow[t]{2}{*}{ Treatment of pre-formed biofilm } & $\mathrm{S}$ & 3.35 & 7.18 & 10.21 & 12.35 & 5.52 & & \\
\hline & $\mathrm{R}$ & 1.56 & 9.69 & 7.20 & 2.77 & 8.00 & & \\
\hline
\end{tabular}

Notes: Data are expressed in percentage on the total biofilm biomass. S, methicillin susceptible $S$. aureus or carbapenems susceptible $P$. aeruginosa, R, methicillin resistant $S$. aureus or carbapenems resistant $P$. aeruginosa.

Abbreviations: CQ, chlorquinaldol; GEN, gentamicin; FA, fusidic acid.

action, which could possibly be a metal-binding site of bacterial enzymes. In the example, $8 \mathrm{HQ}$ was found to inhibit Escherichia coli RNA polymerase activity by chelating the dissociable cations $\mathrm{Mn}^{2+}$ and $\mathrm{Mg}^{2+}{ }^{42}$ Thus, CQ might inhibit pyocyanin production by interfering with PhzE, a $\mathrm{Mg}^{2+}$-dependent enzyme that catalyzes the first

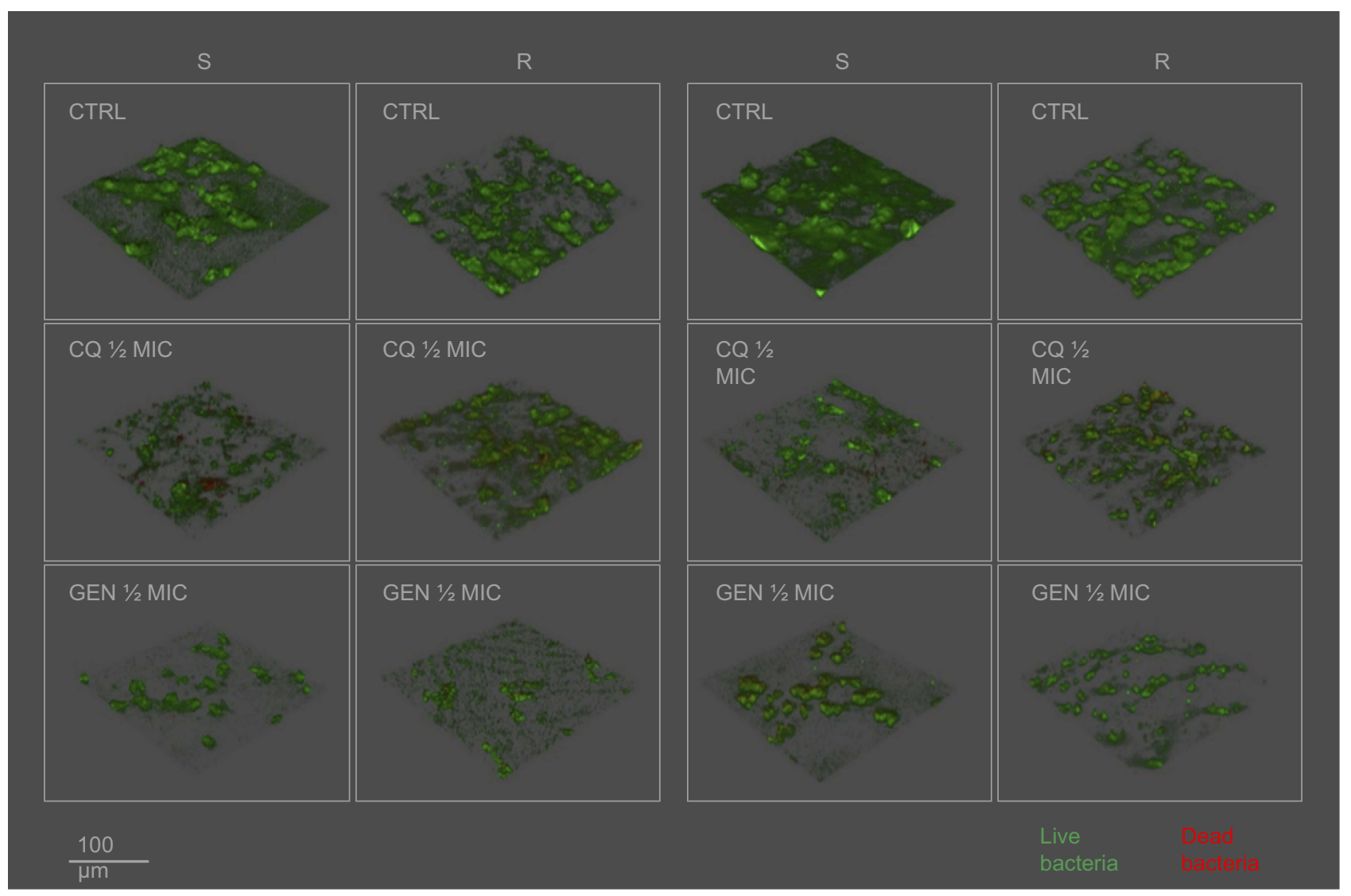

Figure 5 Three-dimensional reconstruction of $P$. aeruginosa biofilm analyzed by means of confocal laser scan microscopy. 
step in the biosynthesis of pyocyanin and other $P$. aeruginosa phenazines. ${ }^{44}$

All $P$. aeruginosa-resistant strains used in this study possess an "impermeability to carbapenems" phenotype. This is usually due to a mutation or downregulation event of the gene coding for OprD porin, which selectively confer resistance to imipenem and meropenem. ${ }^{45}$ However, such information refers to tests performed on planktonic cells, while such adaptations might be more aspecific and may reveal other phenotypes when tested on biofilms. GEN displayed scarce efficacy against $P$. aeruginosa biofilm, with significant reductions mostly when highest concentrations were applied (Figures $1 C, 2 \mathrm{C}$ and 2D). Since no differences were observed between susceptible and resistant strains when tested in the planktonic form (Table 1), a reason for the failure to prevent or eradicate MRSA and carbapenems resistant $P$. aeruginosa strains biofilm should be addressed to global changes in the regulation, either reflecting different matrix composition or altered expression on the bacterial surface.

A limitation of this study is that a combination of the different compound was not tested. Indeed, anti-biofilm therapy should point simultaneously to multiple targets. Enhanced antibiofilm activity was reported when traditional antibiotics were used in combination with alternative antimicrobials, such as metal ions chelation and virulence attenuation approaches. ${ }^{46,47}$ Indeed, aspecific activity might be at the basis of limiting chemoresistance outbreaks as already observed for CQ. Whereas multiple passages of $S$. aureus in presence of gentamicin, bacitracin and neomycin led to a marked increase in MIC concentrations, the sensitivity to $\mathrm{CQ}$ remained largely unchanged. ${ }^{48}$ Furthermore, minimum biofilm eliminating concentration (MBEC) was not investigated in this study. Such assay, performed by means of the Calgary device, would have provided important information on the extent of the contribution of such antibiotic resistance determinants to the increase of biofilm inhibitory concentrations.

\section{Conclusion}

In conclusion, the observations made in this study strengthen the need to expand the tests routinely performed on antimicrobials to biofilm bacteria and to include in the tests pathogenic isolates of the same species with different resistance patterns, since acquired genes and mutations might reflect in global changes that are not identifiable in the planktonic state. Furthermore, as biofilm recalcitrance to antibiotics is based on more than one mechanism of resistance, alternative methods for biofilm prevention and/or eradication should be considered.

\section{Acknowledgments}

This work was supported by a grant from Bayer (Milan, Italy). The sponsor was not involved in study design, in the collection, analysis and interpretation of data, the writing of the report nor in the decision to submit the article for publication.

\section{Author contributions}

All authors contributed to data analysis, drafting or revising the article, gave final approval of the version to be published, and agree to be accountable for all aspects of the work.

\section{Disclosure}

Dr Elena De Vecchi report grants from Bayer SpA, Milan Italy, during the conduct of the study. The authors report no other conflicts of interest in this work.

\section{References}

1. Newton H, Edwards J, Mitchell L, Percival SL. Role of slough and biofilm in delaying healing in chronic wounds. Br J Nurs. 2017;26 (Sup20a):S4-S11. doi:10.12968/bjon.2017.26.Sup20a.S4

2. Hall-Stoodley L, Costerton JW, Stoodley P. Bacterial biofilms: from the natural environment to infectious diseases. Nat Rev Microbiol. 2004;2:95-108. doi:10.1038/nrmicro821

3. James GA, Swogger E, Wolcott R, et al. Biofilms in chronic wounds. Wound Repair Regen. 2008;16:37-44. doi:10.1111/j.1524475X.2007.00321.x

4. Malone M, Goeres DM, Gosbell I, Vickery K, Jensen S, Stoodley P. Approaches to biofilm-associated infections: the need for standardized and relevant biofilm methods for clinical applications. Expert Rev Anti Infect Ther. 2017;15(2):147-156. doi:10.1080/14787210.2017.1262257

5. Hall-Stoodley L, Stoodley P. Evolving concepts in biofilm infections. Cell Microbiol. 2009;11:1034-1043. doi:10.1111/j.1462-5822.2009.01323.x

6. Høiby N, Bjarnsholt T, Moser C. ESCMID guideline for the diagnosis and treatment of biofilm infections 2014. Clin Microbiol Infect. 2015;21(Suppl 1):S1-S25. doi:10.1016/j.cmi.2014.10.024

7. Heng YK, Tan KT, Sen P, et al. Staphylococcus aureus and topical fusidic acid use: results of a clinical audit on antimicrobial resistance. Int $J$ Dermatol. 2013;52:876-881. doi:10.1111/j.1365-4632.2012.05747.x

8. Bortolin M, Bidossi A, De Vecchi E, Avveniente M, Drago L. In vitro antimicrobial activity of chlorquinaldol against microorganisms responsible for skin and soft tissue infections: comparative evaluation with gentamicin and fusidic acid. Front Microbiol. 2017;8(8):1039. doi:10.3389/fmicb.2017.01039

9. Maeder E, Schindléry C, Macarol V, Schoenenberger PM. A comparative multicenter trial of halometasone/triclosan cream and diflucortolone valerate/chlorquinaldol cream in the treatment of acute dermatomycoses. $J$ Int Med Res. 1983;11(Suppl 1):48-52.

10. Hoppe G. Diflucortolone valerate. Asian experience. Drugs. 1988;36 (Suppl 5):24-33. doi:10.2165/00003495-198800365-00006

11. Corrihons I, Dutilh B, Bébéar C. In vitro activity of an antiseptic, chlorquinaldol, against Neisseria gonorrhoeae and Chlamydia trachomatis. Pathol Biol. 1991;39(2):136-139.

12. Prachayasittikul V, Prachayasittikul S, Ruchirawat S, Prachayasittikul V. 8-Hydroxyquinolines: a review of their metal chelating properties and medicinal applications. Drug Des Devel Ther. 2013;7:11571178. doi:10.2147/DDDT.S49763

13. Littman ML. Antimycotic effect of chlorquinaldol. Trans N Y Acad Sci. $1955 ; 18: 161-174$. 
14. Mann PH, Fratta I, Sigg EB. Susceptibility testing of 200 strains of Staphylococcus aureus to chlorquinaldol. Antibiot Chemother. 1960;10:771-772.

15. Clinical and Laboratory Standards Institute guidelines (CLSI). Methods for Dilution Antimicrobial Susceptibility Tests for Bacteria that Grow Aerobically. CLSI. 2015; 10th ed.

16. Christensen GD, Simpson WA, Younger JJ, et al. Adherence of coagulase-negative staphylococci to plastic tissue culture plates: a quantitative model for the adherence of staphylococci to medical devices. J Clin Microbiol. 1985;22:996-1006.

17. Meyer JM, Abdallah MA. The fluorescent pigment of pseudomonas fluorescens: biosynthesis, purification and physicochemical properties. Microbiology. 1999;107:319-328.

18. Reszka KJ, O’Malley Y, McCormick ML, Denning GM, Britigan BE. Oxidation of pyocyanin, a cytotoxic product from Pseudomonas aeruginosa, by microperoxidase 11 and hydrogen peroxide. Free Radic Biol Med. 2004;36(11):1448-1459. doi:10.1016/j.freeradbiomed.2004.03.011

19. Abu EA, Su S, Sallans L, et al. Cyclic voltammetric, fluorescence and biological analysis of purified aeruginosin $\mathrm{A}$, a secreted red pigment of Pseudomonas aeruginosa PAO1. Microbiology. 2013;159(Pt 8):1736-1747. doi:10.1099/mic.0.065235-0

20. Lin YT, Wang CT, Chiang BL. Role of bacterial pathogens in atopic dermatitis. Clin Rev Allergy Immunol. 2007;33(3):167-177. doi:10.1007/s12016-007-0044-5

21. Sonesson A, Przybyszewska K, Eriksson S, et al. Identification of bacterial biofilm and the Staphylococcus aureus derived protease, staphopain, on the skin surface of patients with atopic dermatitis. Sci Rep. 2017;7(1):8689. doi:10.1038/s41598-017-08046-2

22. Akiyama H, Hamada T, Huh WK, et al. Confocal laser scanning microscopic observation of glycocalyx production by Staphylococcus aureus in skin lesions of bullous impetigo, atopic dermatitis and pemphigus foliaceus. Br J Dermatol. 2003;148(3):526-532.

23. Bergen PJ, Landersdorfer CB, Lee HJ, Li J, Nation RL. 'Old' antibiotics for emerging multidrug-resistant bacteria. Curr Opin Infect Dis. 2012;25(6):626-633. doi:10.1097/QCO.0b013e328358afe5

24. Strukelj R, Manfredi B, Finelli F. Trattamento di dermopatie corticoidosensibili infette con l'associazione diflucortolone valeratoclorchinaldolo. Giornale Italiano Di Ricerche Cliniche $E$ Terapeutiche. 1982;3:177-186.

25. Agner T, Menné T. Sensitivity to clioquinol and chlorquinaldol in the quinoline mix. Contact Dermatitis. 1993;29(3):163. doi:10.1111/ j.1600-0536.1993.tb03524.x

26. Meyer-Rohn J, Puschmann M. In vitro demonstration of the antibacterial and antimycotic efficacy of a preparation containing nystatin and chlorquinaldol compared with similar antimicrobial agents. Mykosen. 1980;23:320-324.

27. Niu H, Yee R, Cui P, et al. Identification of agents active against methicillin-resistant Staphylococcus aureus USA300 from a clinical compound library. Pathogens. 2017;6:3. doi:10.3390/pathogens6030044

28. Hausner M, Wuertz S. High rates of conjugation in bacterial biofilms as determined by quantitative in situ analysis. Appl Environ Microbiol. 1999;65(8):3710-3713.

29. Molin S, Tolker-Nielsen T. Gene transfer occurs with enhanced efficiency in biofilms and induces enhanced stabilisation of the biofilm structure. Curr Opin Biotechnol. 2003;14(3):255-261.

30. Madsen JS, Burmølle M, Hansen LH, Sørensen SJ. The interconnection between biofilm formation and horizontal gene transfer. FEMS Immunol Med Microbiol. 2012;65(2):183-195. doi:10.1111/j.1574695X.2012.00960.x

31. Savage VJ, Chopra I, O’Neill AJ. Staphylococcus aureus biofilms promote horizontal transfer of antibiotic resistance. Antimicrob Agents Chemother. 2013;57(4):1968-1970. doi:10.1128/AAC.02008-12
32. Driffield K, Miller K, Bostock JM, O’Neill AJ, Chopra I. Increased mutability of Pseudomonas aeruginosa in biofilms. J Antimicrob Chemother. 2008;61(5):1053-1056. doi:10.1093/jac/dkn044

33. Boles BR, Singh PK. Endogenous oxidative stress produces diversity and adaptability in biofilm communities. Proc Natl Acad Sci USA. 2008;105(34):12503-12508. doi:10.1073/pnas.0801499105

34. Williamson DA, Monecke S, Heffernan $\mathrm{H}$, et al. High usage of topical fusidic acid and rapid clonal expansion of fusidic acid-resistant Staphylococcus aureus: a cautionary tale. Clin Infect Dis. 2014;59:1451-1454. doi:10.1093/cid/ciu658

35. McCarthy H, Rudkin JK, Black NS, Gallagher L, O'Neill E, O'Gara JP. Methicillin resistance and the biofilm phenotype in Staphylococcus aureus. Front Cell Infect Microbiol. 2015;28(5):1.

36. O’Neill E, Pozzi C, Houston P, et al. Association between methicillin susceptibility and biofilm regulation in Staphylococcus aureus isolates from device-related infections. $J$ Clin Microbiol. 2007;45 (5):1379-1388. doi:10.1128/JCM.02280-06

37. Houston P, Rowe SE, Pozzi C, Waters EM, O'Gara JP. Essential role for the major autolysin in the fibronectin-binding protein-mediated Staphylococcus aureus biofilm phenotype. Infect Immun. 2011;79 (3):1153-1165. doi:10.1128/IAI.00364-10

38. Costerton JW. Introduction to biofilm. Int $J$ Antimicrob Agents. 1999;11(3-4):217-21; discussion 237-9.

39. Visca P, Imperi F, Lamont IL. Pyoverdine siderophores: from biogenesis to biosignificance. Trends Microbiol. 2007;15(1):22-30. doi:10.1016/j.tim.2006.11.004

40. Dietrich LE, Price-Whelan A, Petersen A, Whiteley M, Newman DK. The phenazine pyocyanin is a terminal signalling factor in the quorum sensing network of Pseudomonas aeruginosa. Mol Microbiol. 2006;61 (5):1308-1321. doi:10.1111/j.1365-2958.2006.05306.x

41. Das T, Kutty SK, Tavallaie R, et al. Phenazine virulence factor binding to extracellular DNA is important for Pseudomonas aeruginosa biofilm formation. Sci Rep. 2015;11(5):8398. doi:10.1038/ srep08398

42. Fraser RS, Creanor J. The mechanism of inhibition of ribonucleic acid synthesis by 8 -hydroxyquinoline and the antibiotic lomofungin. Biochem J. 1975;147:401-410. doi:10.1042/bj1470401

43. Short BR, Vargas MA, Thomas JC, O'Hanlon S, Enright MC. In vitro activity of a novel compound, the metal ion chelating agent $\mathrm{AQ}^{+}$, against clinical isolates of Staphylococcus aureus. J Antimicrob Chemother. 2006;57(1):104-109. doi:10.1093/jac/dki428

44. Culbertson JE, Toney MD. Expression and characterization of PhzE from $P$. aeruginosa PAO1: aminodeoxyisochorismate synthase involved in pyocyanin and phenazine-1-carboxylate production. Biochim Biophys Acta. 2013;1834(1):240-246. doi:10.1016/j. bbapap.2012.10.010

45. Meletis G, Exindari M, Vavatsi N, Sofianou D, Diza E. Mechanisms responsible for the emergence of carbapenem resistance in Pseudomonas aeruginosa. Hippokratia. 2012;16(4):303-307.

46. Algburi A, Comito N, Kashtanov D, Dicks LM, Chikindas ML. Control of biofilm formation: antibiotics and beyond. Appl Environ Microbiol. 2017;83(3):e02508-e02516. doi:10.1128/AEM.02508-16

47. Banin E, Brady KM, Greenberg EP. Chelator-induced dispersal and killing of Pseudomonas aeruginosa cells in a biofilm. Appl Environ Microbiol. 2006;72(3):2064-2069. doi:10.1128/AEM.72.3.20642069.2006

48. Kessler HJ. Local antiseptics versus antibiotics in topical therapy - the emergence of microbial resistance. Mykosen. 1980;23 (6):285-289. 


\section{Supplementary material}

Table SI Resistance profile of $S$. aureus and $P$. aeruginosa strains used in this study

\begin{tabular}{|l|l|}
\hline Strains & Resistance profile \\
\hline Sau I (S) & PEN, CLI, ERY \\
Sau 2 (S) & PEN \\
Sau $3(S)$ & PEN \\
Sau 4 (S) & PEN, TET \\
Sau $5($ (S) & PEN, RIF \\
Sau 6 (R) & PEN, OXA, LVX \\
Sau $7(R)$ & PEN, OXA, LVX, CLI \\
Sau 8 (R) & PEN, OXA, LVX \\
Sau 9 (R) & PEN, OXA, LVX, CLI, ERY, TET, RIF \\
Sau I0 (R) & PEN, OXA, LVX, ERY, RIF \\
Pae I (S) & AMC, CRO, SXT \\
Pae 2 (S) & AMC, TZP, FEP, SXT \\
Pae 3 (S) & AMC, CRO, SXT \\
Pae 4 (S) & AMC, CFZ, CRO, CIP, LVX, TGC, CAZ \\
Pae 5 (S) & AMC, CFZ, CRO, CIP, LVX, TGC, SXT \\
Pae 6 (R) & AMC, MEM, IPM, SXT, NIT \\
Pae 7 (R) & AMC, TZP, MEM, IPM, TGC, SXT \\
Pae 8 (R) & AMC, CFZ, CRO, MEM, IPM, TGC, SXT \\
Pae 9 (R) & AMC, CFZ, CRO, MEM, IPM, TGC, AMK, SXT \\
Pae I0 (R) & AMC, TZP, CFZ, CRO, CAZ, FEP, MEM, IPM, TGC, SXT \\
\hline
\end{tabular}

Abbreviations: S, MSSA or P. aeruginosa susceptible to carbapenems; R, MRSA or P. aeruginosa resistant to carbapenems; PEN, Penicillin G; CLI, Clindamycin; ERY, Erythromycin; TET, Tetracycline; RIF, Rifampicin; OXA, Oxacillin; LVX, Levofloxacin; AMC, Amoxicillin/clavulanic acid; CRO, Ceftriaxone; FEP, Cefepime; CFZ, Cefazolin; CAZ, Ceftazidime; SXT, Trimethoprim/Sulfamethoxazole; TZP, piperacillina/tazobactam; CIP, Cipro; TGC, Tigeciclin; MEM, Meropenem; IPM, Imipenem; AMK, Amikacin; NIT, Nitrofurantoin.

\section{Publish your work in this journal}

Infection and Drug Resistance is an international, peer-reviewed openaccess journal that focuses on the optimal treatment of infection (bacterial, fungal and viral) and the development and institution of preventive strategies to minimize the development and spread of resistance. The journal is specifically concerned with the epidemiology of antibiotic resistance and the mechanisms of resistance development and diffusion in both hospitals and the community. The manuscript management system is completely online and includes a very quick and fair peerreview system, which is all easy to use. Visit http://www.dovepress.com/ testimonials.php to read real quotes from published authors. 